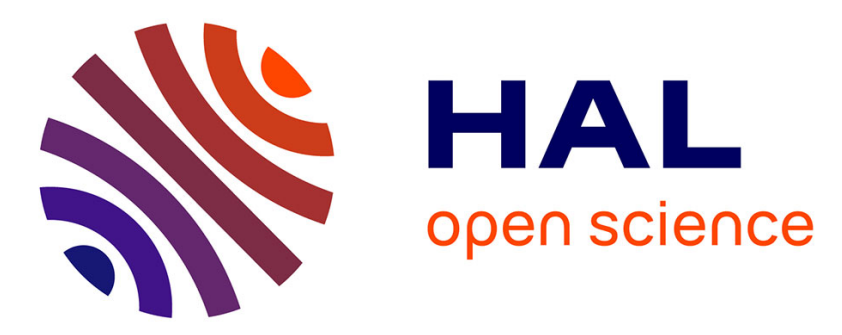

\title{
Cavitation in a liquid-filled cavity surrounded by an elastic medium: Intercoupling of cavitation events in neighboring cavities
}

\author{
Alexander Doinikov, Benjamin Dollet, Philippe Marmottant
}

\section{To cite this version:}

Alexander Doinikov, Benjamin Dollet, Philippe Marmottant. Cavitation in a liquid-filled cavity surrounded by an elastic medium: Intercoupling of cavitation events in neighboring cavities. Physical Review E , 2018, 98 (1), pp.013108. 10.1103/physreve.98.013108 . hal-01906092

\section{HAL Id: hal-01906092 \\ https://hal.science/hal-01906092}

Submitted on 26 Oct 2018

HAL is a multi-disciplinary open access archive for the deposit and dissemination of scientific research documents, whether they are published or not. The documents may come from teaching and research institutions in France or abroad, or from public or private research centers.
L'archive ouverte pluridisciplinaire HAL, est destinée au dépôt et à la diffusion de documents scientifiques de niveau recherche, publiés ou non, émanant des établissements d'enseignement et de recherche français ou étrangers, des laboratoires publics ou privés. 


\title{
Cavitation in a liquid-filled cavity surrounded by an elastic medium: Intercoupling of cavitation events in neighboring cavities
}

\author{
Alexander A. Doinikov, Benjamin Dollet, and Philippe Marmottant \\ LIPhy UMR 5588, CNRS/Université Grenoble-Alpes, Grenoble F-38401, France
}

(Received 28 February 2018; published 27 July 2018)

\begin{abstract}
The subject of the present theoretical study is the dynamics of a cavitation bubble in a spherical liquid-filled cavity surrounded by an infinite elastic solid. Two objectives are pursued. The first is to derive equations for the velocity and pressure fields throughout the liquid filling the cavity and equations for the stress and strain fields throughout the solid medium surrounding the cavity. This derivation is based on the results of our previous paper [A. A. Doinikov et al., Phys. Rev. E 97, 013108 (2018)], where equations for the evolution of a bubble inside a cavity were derived. The second objective is to apply the equations obtained at the first step of the study to ascertain if the cavitation process in one cavity can trigger the nucleation in a neighboring cavity. To this end, we consider a neighboring cavity in which a cavitation bubble is absent. We derive equations that describe the disturbance of the liquid pressure inside the second cavity, assuming this disturbance to be caused by the cavitation process in the first cavity. The developed theory is then used to perform numerical simulations. The results of the simulations show that the magnitude of the background negative pressure inside the second cavity increases at the second half period of the pressure disturbance, which in turn enhances the probability of nucleation in the second cavity.
\end{abstract}

DOI: 10.1103/PhysRevE.98.013108

\section{INTRODUCTION}

Theoretical studies on cavitation phenomena in a microscopic confinement, i.e., in liquid microinclusions inside a solid medium, have received increased attention relatively recently. Such situations, however, occur quite often in nature and technology. Examples can be found in many areas such as geology [1,2], processes in porous media [3-5], tensiometer measurements [6], biology [7-10], etc.

Our interest in this research was inspired by cavitation events that are observed inside trees [9,11-13]. Evaporation processes result in high negative pressures inside water-filled tree conduits $[6,14,15]$, which in turn give rise to the nucleation of cavitation bubbles inside conduits. The bubbles grow, undergo transient oscillations, and emit acoustic waves in the ultrasonic range $[7,16]$. This process has a great influence on tree physiology $[8,10,17,18]$.

Our previous paper [19] provides a detailed review of theoretical models developed to describe cavitation inside trees $[6,14,15,20]$. In the context of the current research, the most important point is that none of the above models proposes equations for the velocity and pressure fields throughout the liquid and equations for the stress and strain fields throughout the solid. The only model that describes the propagation of acoustic waves in the liquid and in the solid is that of Drysdale et al. [21]. However, they use a linear approximation that is valid only at the final stage of the bubble evolution, when the cavitation bubble in the process of growth reaches a final equilibrium radius and undergoes small-amplitude oscillations about this radius.

None of the available models, including that of Drysdale et al. [21], considers the intercoupling of cavitation events in neighboring cavities. All of them are restricted to the analysis of the bubble dynamics in a single cavity. They do not consider the wave propagation throughout the solid (see the clause about Ref. [21] above) and hence their results cannot be used to gain an insight into how the cavitation process in one cavity affects a cavitation event in a neighboring cavity.

The above-stated unsolved problems defined the aims of our study. In our previous paper [19], we derived equations for the growth and the oscillation of a cavitation bubble in a single spherical cavity filled with a liquid and enclosed in an infinite elastic medium. These equations allow one to calculate the variation of the bubble and the cavity radii from a moment that an initial cavitation microbubble appears in the cavity up to a moment that the bubble reaches a final equilibrium radius and undergoes a damped oscillation about this radius. In the present study, we use these results in order to derive equations for the velocity and pressure fields throughout the liquid filling the cavity and equations for the stress and strain fields throughout the solid medium surrounding the cavity. Then, we use the above equations in order to get a notion of how the cavitation process in the cavity affects the possibility of nucleation in a neighboring cavity.

\section{THEORETICAL CALCULATIONS}

The object of our interest is a cavitation bubble in a spherical cavity filled with a compressible viscous liquid and surrounded by an infinite elastic solid; see Fig. 1. It is assumed that the bubble arises due to a high initial negative pressure in the liquid. The bubble then grows and undergoes a damped oscillation until its radius reaches an equilibrium value. The bubble growth leads to the relaxation of the pressure in the liquid and the stress in the solid. The bubble oscillation generates acoustic waves, which propagate through the liquid, penetrate into the 


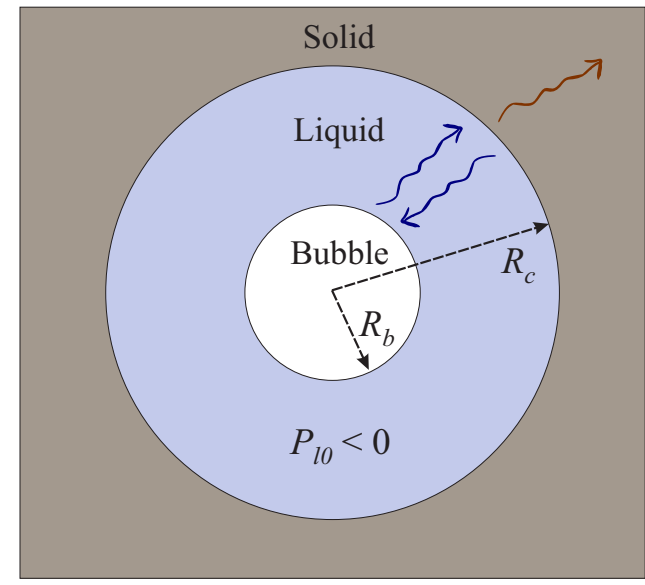

FIG. 1. Geometry of the system under study. A cavitation bubble arises in a liquid-filled cavity due to a high initial negative pressure $P_{l 0}$ in the liquid. The bubble grows and undergoes a damped oscillation until its radius reaches an equilibrium value. The bubble growth leads to the relaxation of the pressure in the liquid and the stress in the solid. The bubble oscillation generates acoustic waves, which propagate through the liquid, penetrate into the solid, and go away.

solid, and go away. The purpose of Sec. II A is to derive equations for the motion that is produced by the relaxation process and the acoustic waves throughout the liquid and the solid. In Sec. II B, we assume that there is a neighboring cavity in which a cavitation bubble is absent. We derive equations that describe the disturbance of the liquid pressure inside the second cavity, assuming this disturbance to be caused by the cavitation process in the first cavity. We use these equations in order to understand how the cavitation process in one cavity can trigger the nucleation in a neighboring cavity. In Sec. III, results of numerical calculations are demonstrated.

\section{A. Equations for a cavity with a bubble}

\section{Evolution of a cavitation bubble}

According to the theory developed in Ref. [19], the evolution of a cavitation bubble in a liquid-filled cavity enclosed in an elastic solid is governed by the following equations:

$$
\begin{aligned}
& \rho_{l 0} R_{b} \ddot{R}_{b}\left(1-\frac{\dot{R}_{b} R_{b}^{3}}{c R_{c}^{3}}-\frac{\dot{R}_{c} R_{b}}{c R_{c}}+\frac{4 \eta_{l} R_{b}}{c \rho_{l 0} R_{c}^{2}}\right) \\
& +\rho_{l 0} \dot{R}_{b}^{2}\left[\frac{3}{2}-\frac{2 \dot{R}_{b} R_{b}^{3}}{c R_{c}^{3}}-\frac{2 \dot{R}_{c} R_{b}}{c R_{c}}\left(1-\frac{R_{b}^{3}}{R_{c}^{3}}\right)\right. \\
& \left.+\frac{4 \eta_{l}}{c \rho_{l 0} R_{b}}\left(1+\frac{2 R_{b}^{2}}{R_{c}^{2}}\right)\right]+\frac{4 \eta_{l} \dot{R}_{b}}{R_{b}}\left(1-\frac{\dot{R}_{b}}{c}-\frac{3 \dot{R}_{c} R_{b}^{3}}{c R_{c}^{3}}\right) \\
& +\frac{2 \sigma_{l}}{R_{b}}-P_{b}-\rho_{l 0} R_{c} \ddot{R}_{c}\left(1-\frac{2 \dot{R}_{c}}{c}+\frac{4 \eta_{l}}{c \rho_{l 0} R_{c}}\right) \\
& -\rho_{l 0} \dot{R}_{c}^{2}\left(\frac{3}{2}-\frac{2 \dot{R}_{c}}{c}\right)-\frac{4 \eta_{l} \dot{R}_{c}}{R_{c}}\left(1-\frac{\dot{R}_{c}}{c}\right)=\tau_{r r}\left(R_{c}, t\right), \\
& R_{b}^{2} \dot{R}_{b}=R_{c}^{2} \dot{R}_{c},
\end{aligned}
$$

$$
\begin{gathered}
\tau_{r r}\left(R_{c}, t\right)=\tau_{0}(t)+\tau_{2}(t)+\tau_{3}(t), \\
\tau_{0}(t)=\left[\frac{(\lambda+2 \mu) P_{\infty}}{\lambda+2 \mu / 3}+4 \mu\right]\left(\frac{R_{c 0}}{R_{c}}-1\right)-\frac{R_{c 0} P_{l 0}}{R_{c}}, \\
\tau_{2}(t)=-\frac{\lambda+2 \mu}{c_{s}^{2}}\left(R_{c} \ddot{R}_{c}+2 \dot{R}_{c}^{2}\right), \\
\quad \times \frac{\left.\rho_{s}(t)=\frac{c_{s} \rho_{l 0}\left[R_{c}-R_{b}\left(1-\rho_{s} / \rho_{l 0}\right)\right]}{\lambda+2 \mu / 3}\right) \frac{R_{b} R_{c 0} \dot{R}_{c}}{R_{c}}}{} \\
\left.+\frac{2 \sigma_{l} R_{c}^{3} \dot{R}_{c}}{R_{b}^{3}}-\frac{4 \eta_{l}\left(R_{c}^{3}-R_{b}^{3}\right) \ddot{R}_{c}}{R_{b}^{2}}+R_{b} R_{c} \dot{P}_{b}\right],
\end{gathered}
$$

where $R_{b}$ and $R_{c}$ are the time-varying radii of the bubble and the cavity, respectively; $\rho_{l 0}$ is the equilibrium liquid density; $c$ is the speed of sound in the liquid; $\eta_{l}$ is the liquid shear viscosity; $\sigma_{l}$ is the surface tension; $P_{b}$ is the pressure within the bubble; $\tau_{r r}\left(R_{c}, t\right)$ is the normal stress in the solid at the cavity surface; $\lambda$ and $\mu$ are the Lamé coefficients; $P_{\infty}$ is the pressure at infinity; $R_{c 0}=R_{c}(0)$ is the initial radius of the cavity; $P_{l 0}$ is the initial negative pressure in the liquid; $c_{s}=\sqrt{(\lambda+2 \mu) / \rho_{s}}$ is the longitudinal wave speed in the solid; $\rho_{s}$ is the equilibrium density of the solid; and the overdot denotes the time derivative.

Equation (1) describes the time evolution of the bubble radius $R_{b}$, Eq. (2) sets a relation between $R_{b}$ and $R_{c}$, and Eqs. (3)-(6) show how to calculate the quantity $\tau_{r r}\left(R_{c}, t\right)$ on the right-hand side of Eq. (1). Note that Eq. (2) appears as if it were derived for an incompressible liquid. However, it was shown in Ref. [19] that this equation remains valid for a compressible liquid as well if we keep to the first order in $1 / c$ because the liquid compressibility manifests itself only in the order $1 / c^{2}$.

\section{Velocity and pressure fields in the liquid}

The theory developed in Ref. [19] gives the following equations for the velocity and the normal stress in the liquid:

$$
\begin{aligned}
v(r, t)= & \frac{f(t-r / c)+g(t+r / c)}{r^{2}} \\
& +\frac{f^{\prime}(t-r / c)-g^{\prime}(t+r / c)}{c r}, \\
\sigma_{r r}(r, t)= & -\frac{\rho_{l 0}}{r}\left[f^{\prime}(t-r / c)+g^{\prime}(t+r / c)\right] \\
& +\frac{\rho_{l 0}}{2} v^{2}(r, t)-\frac{4 \eta_{l}}{r} v(r, t),
\end{aligned}
$$

where $r$ is the radial coordinate measured from the center of the cavity and the prime denotes the derivative with respect to the argument in brackets.

The derivation performed in Ref. [19] did not require the calculation of the functions $f(t-r / c)$ and $g(t+r / c)$ at arbitrary $r$. Expressions for the above functions at the surfaces of the bubble and the cavity were only necessary. However, the calculation of the velocity and pressure fields throughout the liquid requires a knowledge of $f(t-r / c)$ and $g(t+r / c)$ at arbitrary $r$. 
To evaluate $f(t-r / c)$ and $g(t+r / c)$, let us expand them into a Taylor series as follows:

$$
\begin{aligned}
f(t-r / c)= & f\left[t-R_{b} / c-\left(r-R_{b}\right) / c\right] \\
\approx & f\left(t-R_{b} / c\right)-\frac{r-R_{b}}{c} f^{\prime}\left(t-R_{b} / c\right) \\
& +O\left(\frac{1}{c^{2}}\right), \\
g(t+r / c)= & g\left[t+R_{b} / c+\left(r-R_{b}\right) / c\right] \\
\approx & g\left(t+R_{b} / c\right)+\frac{r-R_{b}}{c} g^{\prime}\left(t+R_{b} / c\right) \\
& +O\left(\frac{1}{c^{2}}\right) .
\end{aligned}
$$

These equations express $f(t-r / c)$ and $g(t+r / c)$ in terms of their values at the surface of the bubble.

Substitution of Eqs. (9) and (10) into Eq. (7) yields

$$
\begin{aligned}
v(r, t)= & \frac{f\left(t-R_{b} / c\right)+g\left(t+R_{b} / c\right)}{r^{2}} \\
& +\frac{R_{b}\left[f^{\prime}\left(t-R_{b} / c\right)-g^{\prime}\left(t+R_{b} / c\right)\right]}{c r^{2}} .
\end{aligned}
$$

Expanding Eq. (11) into a Taylor series in terms of $R_{b} / c$, one obtains

$$
v(r, t)=\frac{f(t)+g(t)}{r^{2}}+O\left(\frac{1}{c^{2}}\right) .
$$

Equation (12) shows that terms of the order $1 / c$ are absent from the expansion of the liquid velocity in a Taylor series. This means that, with an accuracy up to $1 / c$, the liquid velocity in a compressible liquid is given by the same expression as in an incompressible liquid.

From the boundary condition

$$
v\left(R_{b}, t\right)=\dot{R}_{b}
$$

one gets

$$
f(t)+g(t)=R_{b}^{2} \dot{R}_{b},
$$

so the velocity is given by

$$
v(r, t)=\frac{R_{b}^{2} \dot{R}_{b}}{r^{2}}+O\left(\frac{1}{c^{2}}\right) .
$$

Expanding $f^{\prime}(t-r / c)$ and $g^{\prime}(t+r / c)$ into a Taylor series similar to Eqs. (9) and (10) and substituting them into Eq. (8), one obtains

$$
\begin{aligned}
\sigma_{r r}(r, t)= & -\frac{\rho_{l 0}}{r}\left[f^{\prime}\left(t-R_{b} / c\right)+g^{\prime}\left(t+R_{b} / c\right)\right] \\
& +\frac{\rho_{l 0}}{2} v^{2}(r, t)-\frac{4 \eta_{l}}{r} v(r, t)+\frac{\rho_{l 0}}{c}\left(1-\frac{R_{b}}{r}\right) \\
& \times\left[f^{\prime \prime}\left(t-R_{b} / c\right)-g^{\prime \prime}\left(t+R_{b} / c\right)\right] .
\end{aligned}
$$

Let us apply the boundary conditions for $\sigma_{r r}(r, t)$ [19],

$$
\begin{gathered}
P_{b}=\frac{2 \sigma_{l}}{R_{b}}-\sigma_{r r}\left(R_{b}, t\right), \\
\sigma_{r r}\left(R_{c}, t\right)=\tau_{r r}\left(R_{c}, t\right) .
\end{gathered}
$$

Substitution of Eq. (16) into Eqs. (17) and (18) yields

$$
\begin{aligned}
& \frac{\rho_{l 0}}{R_{b}}\left[f^{\prime}\left(t-R_{b} / c\right)+g^{\prime}\left(t+R_{b} / c\right)\right] \\
&= P_{b}-\frac{2 \sigma_{l}}{R_{b}}+\frac{\rho_{l 0}}{2} \dot{R}_{b}^{2}-\frac{4 \eta_{l}}{R_{b}} \dot{R}_{b}, \\
& \tau_{r r}\left(R_{c}, t\right)=-\frac{\rho_{l 0}}{R_{c}}\left[f^{\prime}\left(t-R_{b} / c\right)+g^{\prime}\left(t+R_{b} / c\right)\right] \\
&+\frac{\rho_{l 0}}{2} \dot{R}_{c}^{2}-\frac{4 \eta_{l}}{R_{c}} \dot{R}_{c}+\frac{\rho_{l 0}}{c}\left(1-\frac{R_{b}}{R_{c}}\right) \\
& \times\left[f^{\prime \prime}\left(t-R_{b} / c\right)-g^{\prime \prime}\left(t+R_{b} / c\right)\right] .
\end{aligned}
$$

From these equations, it follows that

$$
\begin{gathered}
\rho_{l 0}\left[f^{\prime}\left(t-R_{b} / c\right)+g^{\prime}\left(t+R_{b} / c\right)\right] \\
=R_{b} P_{b}-2 \sigma_{l}+\frac{\rho_{l 0} R_{b} \dot{R}_{b}^{2}}{2}-4 \eta_{l} \dot{R}_{b}, \\
\frac{\rho_{l 0}}{c}\left[f^{\prime \prime}\left(t-R_{b} / c\right)-g^{\prime \prime}\left(t+R_{b} / c\right)\right] \\
=\frac{R_{c}}{R_{c}-R_{b}}\left[\tau_{r r}\left(R_{c}, t\right)+\frac{R_{b} P_{b}}{R_{c}}-\frac{2 \sigma_{l}}{R_{c}}\right. \\
\left.+\frac{\rho_{l 0}\left(R_{b} \dot{R}_{b}^{2}-R_{c} \dot{R}_{c}^{2}\right)}{2 R_{c}}+\frac{4 \eta_{l}}{R_{c}}\left(\dot{R}_{c}-\dot{R}_{b}\right)\right] .
\end{gathered}
$$

Substitution of Eqs. (15), (21), and (22) into Eq. (16) yields

$$
\begin{aligned}
\sigma_{r r}(r, t)= & -\frac{1}{r}\left(R_{b} P_{b}-2 \sigma_{l}+\frac{\rho_{l 0} R_{b} \dot{R}_{b}^{2}}{2}-4 \eta_{l} \dot{R}_{b}\right) \\
& +\frac{\rho_{l 0} R_{b}^{4} \dot{R}_{b}^{2}}{2 r^{4}}-\frac{4 \eta_{l} R_{b}^{2} \dot{R}_{b}}{r^{3}} \\
& +\frac{\left(r-R_{b}\right) R_{c}}{r\left(R_{c}-R_{b}\right)}\left[\tau_{r r}\left(R_{c}, t\right)+\frac{R_{b} P_{b}}{R_{c}}-\frac{2 \sigma_{l}}{R_{c}}\right. \\
& \left.+\frac{\rho_{l 0}\left(R_{b} \dot{R}_{b}^{2}-R_{c} \dot{R}_{c}^{2}\right)}{2 R_{c}}+\frac{4 \eta_{l}}{R_{c}}\left(\dot{R}_{c}-\dot{R}_{b}\right)\right] .
\end{aligned}
$$

The liquid pressure, with an accuracy up to $1 / c$, is given by

$$
p(r, t)=-\sigma_{r r}(r, t)-\frac{4 \eta_{l}}{r} v(r, t) .
$$

Substituting Eq. (23) into Eq. (24), one obtains

$$
\begin{aligned}
p(r, t)= & \frac{1}{r}\left(R_{b} P_{b}-2 \sigma_{l}+\frac{\rho_{l 0} R_{b} \dot{R}_{b}^{2}}{2}-4 \eta_{l} \dot{R}_{b}\right)-\frac{\rho_{l 0} R_{b}^{4} \dot{R}_{b}^{2}}{2 r^{4}} \\
& -\frac{\left(r-R_{b}\right) R_{c}}{r\left(R_{c}-R_{b}\right)}\left[\tau_{r r}\left(R_{c}, t\right)+\frac{R_{b} P_{b}}{R_{c}}-\frac{2 \sigma_{l}}{R_{c}}\right. \\
& \left.+\frac{\rho_{l 0}\left(R_{b} \dot{R}_{b}^{2}-R_{c} \dot{R}_{c}^{2}\right)}{2 R_{c}}+\frac{4 \eta_{l}}{R_{c}}\left(\dot{R}_{c}-\dot{R}_{b}\right)\right]
\end{aligned}
$$


To sum up, the velocity and the pressure fields in the liquid at any values of $r$ are given by Eqs. (15) and (25). These equations involve the quantities $R_{b}, R_{c}$, and $\tau_{r r}\left(R_{c}, t\right)$. The equations for these quantities, derived in our previous paper [19], are provided in Sec. II A 1.

It should be noted that Eqs. (15) and (25) look as if the liquid were assumed incompressible. This result follows from the fact that the Taylor expansions for the liquid velocity and the liquid pressure do not involve terms of the order $1 / c$. For a gas bubble in an infinite liquid, this mathematical fact was first pointed out by Gilmore [22]. Our derivation shows that in the case of a bubble in a cavity, a similar mathematical effect occurs. Thus, one can state that, with an accuracy up to $1 / c$, both incompressible and compressible liquid models lead to the same expressions for the velocity and the pressure, while compressibility corrections appear only through the equations for $R_{b}, R_{c}$, and $\tau_{r r}\left(R_{c}, t\right)$.

\section{Stress and strain fields in the solid}

In Ref. [19], the following equation for the stress in the solid was derived:

$$
\begin{aligned}
\tau_{r r}(r, t)= & -P_{\infty}-4 \mu\left[\frac{s\left(t-r / c_{s}\right)}{r^{3}}+\frac{s^{\prime}\left(t-r / c_{s}\right)}{c_{s} r^{2}}\right] \\
& -\frac{(\lambda+2 \mu) s^{\prime \prime}\left(t-r / c_{s}\right)}{c_{s}^{2} r}
\end{aligned}
$$

As with the liquid, we need to evaluate $s\left(t-r / c_{s}\right)$ and its derivatives at arbitrary $r$.

Let us expand $s\left(t-r / c_{s}\right)$ into a Taylor series as follows:

$$
\begin{aligned}
s\left(t-r / c_{s}\right)= & s\left[t-R_{c} / c_{s}-\left(r-R_{c}\right) / c_{s}\right] \\
\approx & s\left(t-R_{c} / c_{s}\right)-\frac{r-R_{c}}{c_{s}} s^{\prime}\left(t-R_{c} / c_{s}\right) \\
& +\frac{\left(r-R_{c}\right)^{2}}{2 c_{s}^{2}} s^{\prime \prime}\left(t-R_{c} / c_{s}\right)+O\left(\frac{1}{c_{s}^{3}}\right) .
\end{aligned}
$$

This equation expresses $s\left(t-r / c_{s}\right)$ through its value and the values of its derivatives that are calculated at the surface of the cavity.

Expanding $s^{\prime}\left(t-r / c_{s}\right)$ and $s^{\prime \prime}\left(t-r / c_{s}\right)$ by the same way and substituting them along with Eq. (27) into Eq. (26), one obtains, with an accuracy up to $1 / c_{s}^{2}$,

$$
\begin{aligned}
\tau_{r r}(r, t)= & -P_{\infty}-\frac{4 \mu}{r^{3}}\left[s\left(t-R_{c} / c_{s}\right)+\frac{R_{c}}{c_{s}} s^{\prime}\left(t-R_{c} / c_{s}\right)\right] \\
& -\frac{s^{\prime \prime}\left(t-R_{c} / c_{s}\right)}{c_{s}^{2} r}\left(\lambda+\frac{2 \mu R_{c}^{2}}{r^{2}}\right) .
\end{aligned}
$$

Let us use relations between $s\left(t-R_{c} / c_{s}\right)$ and its derivatives derived in Ref. [19],

$$
\begin{gathered}
\frac{R_{c}}{c_{s}} s^{\prime \prime}\left(t-R_{c} / c_{s}\right)=R_{c}^{2} \dot{R}_{c}-s^{\prime}\left(t-R_{c} / c_{s}\right), \\
s\left(t-R_{c} / c_{s}\right)=R_{c}^{2} u\left(R_{c}, t\right)+\frac{P_{\infty} R_{c}^{3}}{3 \lambda+2 \mu}-\frac{R_{c}}{c_{s}} s^{\prime}\left(t-R_{c} / c_{s}\right),
\end{gathered}
$$

where $u\left(R_{c}, t\right)$ denotes the displacement of the cavity surface, given by

$$
u\left(R_{c}, t\right)=R_{c}-R_{c 0}+\frac{R_{c 0}}{4 \mu}\left(P_{l 0}-\frac{\lambda+2 \mu}{\lambda+2 \mu / 3} P_{\infty}\right)
$$

see Ref. [19].

Substitution of Eqs. (29) and (30) into Eq. (28) yields

$$
\begin{aligned}
\tau_{r r}(r, t)= & -P_{\infty}-\frac{4 \mu}{r^{3}}\left[R_{c}^{2} u\left(R_{c}, t\right)+\frac{P_{\infty} R_{c}^{3}}{3 \lambda+2 \mu}\right] \\
& +\frac{1}{c_{s} r}\left[\frac{s^{\prime}\left(t-R_{c} / c_{s}\right)}{R_{c}}-R_{c} \dot{R}_{c}\right]\left(\lambda+\frac{2 \mu R_{c}^{2}}{r^{2}}\right) .
\end{aligned}
$$

Setting $r=R_{c}$ in Eq. (32), one can represent $s^{\prime}\left(t-R_{c} / c_{s}\right)$ as

$$
\begin{aligned}
s^{\prime}\left(t-R_{c} / c_{s}\right)= & R_{c}^{2}\left(\dot{R}_{c}+\frac{c_{s} P_{\infty}}{\lambda+2 \mu / 3}\right) \\
& +\frac{c_{s} R_{c}\left[4 \mu u\left(R_{c}, t\right)+R_{c} \tau_{r r}\left(R_{c}, t\right)\right]}{\lambda+2 \mu} .
\end{aligned}
$$

Substitution of Eq. (33) into Eq. (32) yields

$$
\begin{aligned}
\tau_{r r}(r, t)= & -P_{\infty}+\frac{P_{\infty} R_{c}}{(\lambda+2 \mu / 3) r}\left(\lambda+\frac{2 \mu R_{c}^{2}}{3 r^{2}}\right) \\
& +\frac{4 \mu \lambda u\left(R_{c}, t\right)}{(\lambda+2 \mu) r}\left(1-\frac{R_{c}^{2}}{r^{2}}\right) \\
& +\frac{R_{c} \tau_{r r}\left(R_{c}, t\right)}{(\lambda+2 \mu) r}\left(\lambda+\frac{2 \mu R_{c}^{2}}{r^{2}}\right) .
\end{aligned}
$$

Finally, substituting Eq. (31) into Eq. (34), after some rearrangement, one obtains

$$
\begin{aligned}
\tau_{r r}(r, t)= & -P_{\infty}+\frac{3 \lambda P_{\infty}}{3 \lambda+2 \mu}\left[\left(1-\frac{R_{c 0}}{R_{c}}\right) \frac{R_{c}}{r}\right. \\
& \left.+\left(\frac{R_{c 0}}{R_{c}}+\frac{2 \mu}{3 \lambda}\right) \frac{R_{c}^{3}}{r^{3}}\right] \\
& +\frac{\lambda P_{l 0}}{\lambda+2 \mu}\left[\frac{R_{c 0}}{R_{c}}+\frac{4 \mu}{P_{l 0}}\left(1-\frac{R_{c 0}}{R_{c}}\right)\right]\left(\frac{R_{c}}{r}-\frac{R_{c}^{3}}{r^{3}}\right) \\
& +\frac{\lambda \tau_{r r}\left(R_{c}, t\right)}{\lambda+2 \mu}\left(\frac{R_{c}}{r}+\frac{2 \mu R_{c}^{3}}{\lambda r^{3}}\right) .
\end{aligned}
$$

This equation expresses the stress in the solid at any $r$ through the values of the stress at $r=R_{c}, \tau_{r r}\left(R_{c}, t\right)$, which can be calculated using the results of our previous paper [19].

A similar derivation allows one to find the strain (displacement) field $u(r, t)$ throughout the solid.

In Ref. [19], the following equation for $u(r, t)$ was derived:

$$
u(r, t)=-\frac{P_{\infty} r}{3 \lambda+2 \mu}+\frac{s\left(t-r / c_{s}\right)}{r^{2}}+\frac{s^{\prime}\left(t-r / c_{s}\right)}{c_{s} r} .
$$

Substituting Eq. (27) for $s\left(t-r / c_{s}\right)$ and a similar expansion for $s^{\prime}\left(t-r / c_{s}\right)$, one obtains, with accuracy up to $1 / c_{s}^{2}$,

$$
\begin{aligned}
u(r, t)= & -\frac{P_{\infty} r}{3 \lambda+2 \mu}+\frac{1}{r^{2}} s\left(t-R_{c} / c_{s}\right)+\frac{R_{c}}{c_{s} r^{2}} s^{\prime}\left(t-R_{c} / c_{s}\right) \\
& -\frac{1}{2 c_{s}^{2}}\left(1-\frac{R_{c}^{2}}{r^{2}}\right) s^{\prime \prime}\left(t-R_{c} / c_{s}\right) .
\end{aligned}
$$


Substitution of Eqs. (29) and (30) gives

$$
\begin{aligned}
u(r, t)= & -\frac{P_{\infty} r}{3 \lambda+2 \mu}\left(1-\frac{R_{c}^{3}}{r^{3}}\right)-\frac{R_{c} \dot{R}_{c}}{2 c_{s}}\left(1-\frac{R_{c}^{2}}{r^{2}}\right) \\
& +\frac{R_{c}^{2} u\left(R_{c}, t\right)}{r^{2}}+\frac{1}{2 c_{s} R_{c}}\left(1-\frac{R_{c}^{2}}{r^{2}}\right) s^{\prime}\left(t-R_{c} / c_{s}\right) .
\end{aligned}
$$

Finally, eliminating $s^{\prime}\left(t-R_{c} / c_{s}\right)$ by Eq. (33), one gets

$$
\begin{aligned}
u(r, t)= & -\frac{P_{\infty}}{3 \lambda+2 \mu}\left(r-\frac{3 R_{c}}{2}+\frac{R_{c}^{3}}{2 r^{2}}\right) \\
& +\frac{u\left(R_{c}, t\right)}{\lambda+2 \mu}\left(2 \mu+\frac{\lambda R_{c}^{2}}{r^{2}}\right) \\
& +\frac{R_{c} \tau_{r r}\left(R_{c}, t\right)}{2(\lambda+2 \mu)}\left(1-\frac{R_{c}^{2}}{r^{2}}\right) .
\end{aligned}
$$

Equation (39) reveals a limitation of the assumption, made in Ref. [19], that the length of the solid is infinite. According to Eq. (39), the displacement at infinity is infinite. This occurs for the following reason. The displacement of a solid is caused by a force that acts on the surface of the solid. The force is calculated as a product of surface area and pressure. In our case of a spherical solid medium, the surface area at infinity is infinite so the force is infinite and hence the displacement is infinite. A similar problem arises when the deformation of a hollow spherical ball is calculated; see Task 2 of Sec. 7 in Ref. [23]. Note, however, that the strain rate, $\partial u / \partial t$, calculated by Eq. (39), is finite.

To sum up, the procedure of the calculation of the stress and the strain at any values of $r$ is the following. We first calculate $R_{b}$ and $R_{c}$ by the equations derived in Ref. [19] and provided in Sec. II A 1. Using the obtained results, we calculate $u\left(R_{c}, t\right)$ from Eq. (31) and $\tau_{r r}\left(R_{c}, t\right)$ from Eqs. (3)-(6). Then we calculate $\tau_{r r}(r, t)$ and $u(r, t)$ from Eqs. (35) and (39).

\section{B. Equations for a cavity without a bubble}

Let us assume that there is another cavity in which a cavitation bubble is yet absent. In what follows, the parameters of the first cavity, which contains a bubble, will be denoted by $r_{1}, R_{c 1}$, and $R_{c 10}$, while the parameters of the second cavity will be denoted by $r_{2}, R_{c 2}$, and $R_{c 20}$; see Fig. 2 .

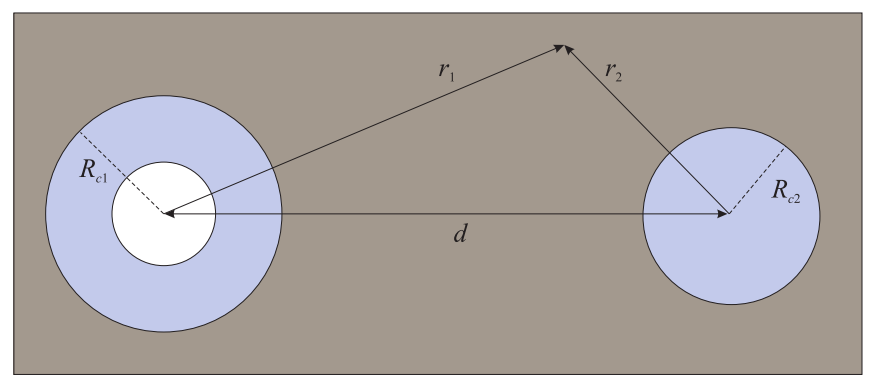

FIG. 2. Two cavities with a separation distance $d$ between their centers. The second (right) cavity does not contain a cavitation bubble and is kept at rest until the disturbance wave from the first (left) cavity reaches it.
Our aim is to calculate the disturbance of the liquid pressure inside the second cavity that is caused by the cavitation process in the first cavity. This knowledge will give an insight into how cavitation in one cavity can trigger the nucleation in a neighboring cavity. We assume that the second cavity is at rest until the disturbance from the first cavity reaches it. With this assumption, we can neglect the influence of the second cavity on the dynamics of the first cavity.

We consider the motion of the liquid in the second cavity in the linear approximation. Numerical calculations presented in Sec. III show that the amplitude of the waves emitted by the first cavity decreases quickly with distance. Therefore, when the disturbance reaches the second cavity, its intensity is relatively low and hence the linear approximation is justified. In this case, the liquid motion inside the second cavity is governed by the following equations [24]:

$$
\begin{gathered}
\frac{\partial \rho_{l 2}}{\partial t}+\rho_{l 20} \nabla \cdot \boldsymbol{v}_{2}=0 \\
\rho_{l 20} \frac{\partial \boldsymbol{v}_{2}}{\partial t}-\eta_{l} \Delta \boldsymbol{v}_{2}-\left(\zeta_{l}+\frac{1}{3} \eta_{l}\right) \nabla\left(\nabla \cdot \boldsymbol{v}_{2}\right)=-\nabla p_{2}, \\
p_{2}=c^{2}\left(\rho_{l 2}-\rho_{l 20}\right),
\end{gathered}
$$

where $\rho_{l 2}$ and $\rho_{l 20}$ are the time-varying liquid density and the liquid density at rest, respectively; $\boldsymbol{v}_{2}$ is the liquid velocity; $\eta_{l}$ and $\zeta_{l}$ are the shear viscosity and the bulk viscosity, respectively; $p_{2}$ is the disturbed liquid pressure; and $c$ is the speed of sound. Note that the values of $\eta_{l}, \zeta_{l}$, and $c$ are assumed to be the same as in the first cavity; i.e., we assume the liquids in both cavities to be identical.

In the case under study, the liquid motion is spherically symmetric and hence irrotational [24]. The velocity of an irrotational flow can be written in terms of a velocity potential, $\varphi_{2}$, as

$$
\boldsymbol{v}_{2}=v_{2}\left(r_{2}, t\right) \boldsymbol{e}_{r 2}=\nabla \varphi_{2}\left(r_{2}, t\right)=\frac{\partial \varphi_{2}}{\partial r_{2}} \boldsymbol{e}_{r 2},
$$

where $\boldsymbol{e}_{r 2}$ is the unit vector along the radial coordinate $r_{2}$. On substitution of Eq. (43), Eqs. (40)-(42) can be recast to

$$
\begin{gathered}
\Delta \varphi_{2}+\frac{1}{c^{2} \rho_{l 20}}\left(\zeta_{l}+\frac{4}{3} \eta_{l}\right) \frac{\partial \Delta \varphi_{2}}{\partial t}-\frac{1}{c^{2}} \frac{\partial^{2} \varphi_{2}}{\partial t^{2}}=0 \\
p_{2}\left(r_{2}, t\right)=-\rho_{l 20} \frac{\partial \varphi_{2}}{\partial t}+\left(\zeta_{l}+\frac{4}{3} \eta_{l}\right) \Delta \varphi_{2}
\end{gathered}
$$

To solve Eq. (44), we use the Fourier transform method [25]. The Fourier transform of $\varphi_{2}\left(r_{2}, t\right)$ is defined by

$$
\hat{\varphi}_{2}\left(r_{2}, \omega\right)=\int_{-\infty}^{+\infty} \varphi_{2}\left(r_{2}, t\right) e^{-i \omega t} d t .
$$

Calculation of the Fourier transform of Eq. (44) yields

$$
\frac{1}{r_{2}^{2}} \frac{\partial}{\partial r_{2}}\left(r_{2}^{2} \frac{\partial \hat{\varphi}_{2}}{\partial r_{2}}\right)+k^{2}(\omega) \hat{\varphi}_{2}=0,
$$

where $k(\omega)$ is given by

$$
k(\omega)=\frac{\omega}{c}\left[1+\frac{i \omega}{c^{2} \rho_{l 20}}\left(\zeta_{l}+\frac{4}{3} \eta_{l}\right)\right]^{-1 / 2}
$$


Equation (47) is known as the spherical Bessel differential equation of zero order $[25,26]$. Its solution is given by

$$
\hat{\varphi}_{2}\left(r_{2}, \omega\right)=A(\omega) j_{0}\left[k(\omega) r_{2}\right],
$$

where $A(\omega)$ is an arbitrary function and $j_{0}(x)$ is the spherical Bessel function of zero order $[25,26]$. Note that Eq. (49) satisfies the condition that the solution must be finite at $r_{2}=0$.

It follows from Eqs. (43) and (46) that the Fourier transform of $v_{2}\left(r_{2}, t\right)$ is calculated by

$$
\hat{v}_{2}\left(r_{2}, \omega\right)=\frac{\partial \hat{\varphi}_{2}}{\partial r_{2}}=-k(\omega) A(\omega) j_{1}\left[k(\omega) r_{2}\right] .
$$

The Fourier transform of $p_{2}\left(r_{2}, t\right)$, making use of Eqs. (47) and (49), is given by

$$
\hat{p}_{2}\left(r_{2}, \omega\right)=-A(\omega) j_{0}\left[k(\omega) r_{2}\right]\left[i \omega \rho_{l 20}+\left(\zeta_{l}+\frac{4}{3} \eta_{l}\right) k^{2}(\omega)\right] .
$$

In order to find $A(\omega)$, we apply the boundary conditions at the surface of the second cavity,

$$
\begin{gathered}
v_{2}\left(r_{2}, t\right)=\frac{\partial u_{2}\left(r_{2}, t\right)}{\partial t} \text { at } r_{2}=R_{c 20}, \\
\sigma_{r r}^{(2)}\left(r_{2}, t\right)=\tau_{r r}^{(1)}\left(r_{1}, t\right)+\tau_{r r}^{(2)}\left(r_{2}, t\right) \text { at } r_{2}=R_{c 20},
\end{gathered}
$$

where $R_{c 20}$ is the equilibrium radius of the second cavity, $u_{2}$ is the displacement produced in the solid by the oscillation of the second cavity, $\sigma_{r r}^{(2)}$ is the liquid normal stress inside the second cavity, and $\tau_{r r}^{(1)}$ and $\tau_{r r}^{(2)}$ are the normal stresses produced in the solid by the first and the second cavities, respectively.

The derivation, as such, carried out in this section is valid at any separations between the cavities. However, when calculating $\tau_{r r}^{(1)}$, we will assume that $d \gg R_{c 10}, R_{c 20}$. In this case, the value of $\tau_{r r}^{(1)}$ at the surface of the second cavity can be approximately calculated by Eq. (35) at $r_{1}=d$.

The liquid normal stress in the second cavity is calculated by [24]

$$
\sigma_{r r}^{(2)}\left(r_{2}, t\right)=-p_{2}+2 \eta_{l} \frac{\partial^{2} \varphi_{2}}{\partial r_{2}^{2}}+\left(\zeta_{l}-\frac{2}{3} \eta_{l}\right) \Delta \varphi_{2} .
$$

By using Eqs. (47), (49), and (51), the Fourier transform of Eq. (54) is defined as

$$
\begin{aligned}
\hat{\sigma}_{r r}^{(2)}\left(r_{2}, \omega\right)= & A(\omega)\left\{i \omega \rho_{l 20} j_{0}\left[k(\omega) r_{2}\right]\right. \\
& \left.+\frac{4 \eta_{l}}{3} k^{2}(\omega)\left[j_{0}\left[k(\omega) r_{2}\right]+j_{2}\left[k(\omega) r_{2}\right]\right]\right\} .
\end{aligned}
$$

Here, we have also used the recurrence formulas for the functions $j_{n}(x)$ [26].

To calculate the Fourier transforms of $u_{2}$ and $\tau_{r r}^{(2)}$, we use the results of our previous paper [19]. According to Eqs. (13) and (15) of Ref. [19], we can write

$$
u_{2}\left(r_{2}, t\right)=\frac{a r_{2}}{3}+\frac{s_{2}\left(t-r_{2} / c_{s}\right)}{r_{2}^{2}}+\frac{s_{2}^{\prime}\left(t-r_{2} / c_{s}\right)}{c_{s} r_{2}},
$$

$$
\begin{aligned}
\tau_{r r}^{(2)}\left(r_{2}, t\right)= & \left(\lambda+\frac{2 \mu}{3}\right) a-4 \mu \\
& \times\left[\frac{s_{2}\left(t-r_{2} / c_{s}\right)}{r_{2}^{3}}+\frac{s_{2}^{\prime}\left(t-r_{2} / c_{s}\right)}{c_{s} r_{2}^{2}}\right] \\
& -\frac{(\lambda+2 \mu) s_{2}^{\prime \prime}\left(t-r_{2} / c_{s}\right)}{c_{s}^{2} r_{2}},
\end{aligned}
$$

where $s_{2}\left(t-r_{2} / c_{s}\right)$ is an unknown function to be determined by the boundary conditions at the cavity surface and $a$ is a constant to be determined by the boundary condition at infinity. For $r_{1,2} \rightarrow \infty, \tau_{r r}^{(2)}+\tau_{r r}^{(1)} \rightarrow-P_{\infty}$, which brings $a=0$.

The Fourier transforms of Eqs. (56) and (57), with $a=0$, are calculated by

$$
\hat{u}_{2}\left(r_{2}, \omega\right)=e^{-i \omega r_{2} / c_{s}} \hat{s}_{2}(\omega)\left(\frac{1}{r_{2}^{2}}+\frac{i \omega}{c_{s} r_{2}}\right),
$$

$\hat{\tau}_{r r}^{(2)}\left(r_{2}, \omega\right)=e^{-i \omega r_{2} / c_{s}} \hat{s}_{2}(\omega)\left[\frac{(\lambda+2 \mu) \omega^{2}}{c_{s}^{2} r_{2}}-\frac{4 \mu}{r_{2}^{3}}-\frac{4 i \mu \omega}{c_{s} r_{2}^{2}}\right]$,

where $\hat{s}_{2}(\omega)$ denotes the Fourier transform of $s_{2}(t)$.

Finally, the Fourier transform of $\tau_{r r}^{(1)}$, denoted by $\hat{\tau}_{r r}^{(1)}\left(r_{1}, \omega\right)$, is calculated by analogy with Eq. (46), using Eq. (35) for $\tau_{r r}^{(1)}$.

Let us now apply the Fourier transform to Eqs. (52) and (53). The result is

$$
\begin{gathered}
\hat{v}_{2}\left(R_{c 20}, \omega\right)=i \omega \hat{u}_{2}\left(R_{c 20}, \omega\right), \\
\hat{\sigma}_{r r}^{(2)}\left(R_{c 20}, \omega\right)=\hat{\tau}_{r r}^{(1)}(d, \omega)+\hat{\tau}_{r r}^{(2)}\left(R_{c 20}, \omega\right) .
\end{gathered}
$$

Substituting Eqs. (50), (55), (58), and (59) into Eqs. (60) and (61), we obtain expressions for $A(\omega)$ and $\hat{s}_{2}(\omega)$ in terms of $\hat{\tau}_{r r}^{(1)}(d, \omega)$,

$$
\begin{aligned}
A(\omega) & =\frac{a_{2} \hat{\tau}_{r r}^{(1)}(d, \omega)}{a_{2} a_{3}-a_{1} a_{4}}, \\
\hat{s}_{2}(\omega) & =\frac{a_{1} \hat{\tau}_{r r}^{(1)}(d, \omega)}{a_{1} a_{4}-a_{2} a_{3}},
\end{aligned}
$$

where

$$
\begin{gathered}
a_{1}=\frac{i k(\omega)}{\omega} j_{1}\left[k(\omega) R_{c 20}\right] \\
a_{2}=-e^{-i \omega R_{c 20} / c_{s}}\left(\frac{1}{R_{c 20}^{2}}+\frac{i \omega}{c_{s} R_{c 20}}\right) \\
a_{3}=i \omega \rho_{l 20} j_{0}\left[k(\omega) R_{c 20}\right]+\frac{4 \eta_{l}}{3} k^{2}(\omega)\left\{j_{0}\left[k(\omega) R_{c 20}\right]\right. \\
\left.+j_{2}\left[k(\omega) R_{c 20}\right]\right\}, \\
a_{4}=e^{-i \omega R_{c 20} / c_{s}}\left[\frac{4 \mu}{R_{c 20}^{3}}+\frac{4 i \mu \omega}{c_{s} R_{c 20}^{2}}-\frac{(\lambda+2 \mu) \omega^{2}}{c_{s}^{2} R_{c 20}}\right]
\end{gathered}
$$

Now that we know $A(\omega)$, we can calculate the liquid pressure inside the second cavity at any values of $r_{2}$ by applying 


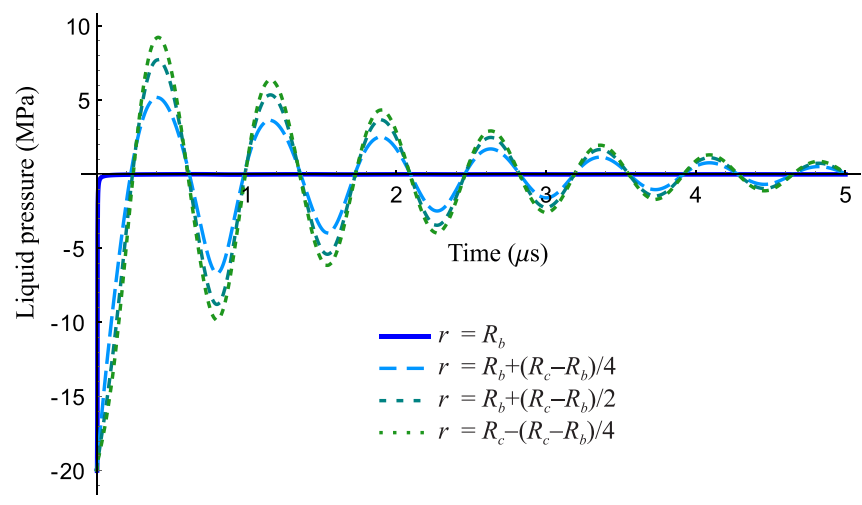

FIG. 3. Liquid pressure as a function of time at different spatial points.

the inverse Fourier transform to Eq. (51),

$$
p_{2}\left(r_{2}, t\right)=\frac{1}{2 \pi} \int_{-\infty}^{+\infty} \hat{p}_{2}\left(r_{2}, \omega\right) e^{i \omega t} d \omega .
$$

\section{NUMERICAL CALCULATIONS}

Numerical calculations were performed at the following values of the physical parameters: $\rho_{l 0}=998 \mathrm{~kg} / \mathrm{m}^{3}, c=1484$ $\mathrm{m} / \mathrm{s}, \eta_{l}=0.001 \mathrm{~Pa} \mathrm{~s}, \sigma_{l}=0.0725 \mathrm{~N} / \mathrm{m}, \rho_{s}=1233 \mathrm{~kg} / \mathrm{m}^{3}$, $\mu=0.74 \mathrm{GPa}, c_{s}=2111 \mathrm{~m} / \mathrm{s}$, and $P_{\infty}=101.3 \mathrm{kPa}$. The parameters of the liquid correspond to water. The parameters of the solid medium were adopted from the paper of Drysdale et al. [21]. They correspond to cavitation experiments on transparent biomimetic wood $[6,14,15]$. It was assumed that there was a vacuum inside the bubbles $\left(P_{b}=0\right)$. An explanation as to why this assumption is relevant is provided in Ref. [21]. In brief, this assumption corresponds to conditions that take place in the case of our main interest, namely, in the case of cavitation events inside trees.

In order to gain an insight into the pressure behavior in the liquid and the stress behavior in the solid, calculations were first made for a single cavity, setting $R_{c 0}=100 \mu \mathrm{m}$ and $P_{l 0}=$ $-20 \mathrm{MPa}$. The results obtained are presented in Figs. 3-7.

Figure 3 illustrates the time behavior of the pressure at different spatial points within the liquid layer. As one can see, the pressure at the bubble wall drops instantaneously to zero as soon as the bubble appears because a vacuum is assumed to be inside the bubble, while the pressure inside the liquid oscillates in response to the oscillation of the bubble.

Figure 4 shows the spatial distribution of the pressure in the liquid layer for different time moments. At $t=0$, the pressure is the same throughout the liquid. When the bubble appears, a strong pressure difference arises between the bubble wall and the cavity surface. This difference is smoothed in time and eventually, when the bubble size reaches an equilibrium value, the pressure again becomes uniform throughout the liquid. For $t \rightarrow \infty$, the pressure tends to $-5.3 \mathrm{kPa}$ throughout the liquid. At this value, as follows from our calculations, an equilibrium is established between the size reached by the bubble in the process of growth, the pressure in the liquid, and the residual stress in the solid. More information on this point can be found in our previous paper [19].

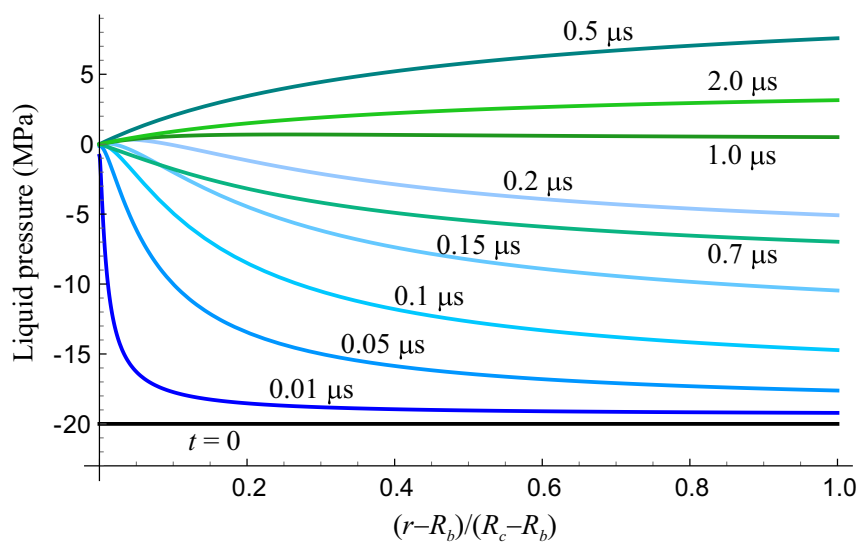

FIG. 4. Spatial distribution of the pressure in the liquid between $R_{b}$ and $R_{c}$ for different time moments. For $t \rightarrow \infty$, the pressure tends to $-5.3 \mathrm{kPa}$ throughout the liquid.

Figure 5 illustrates the time behavior of the normal stress in the solid at different spatial points. As one can see, the stress oscillates and its amplitude decays within a short distance from the cavity surface.

Figure 6 shows the spatial distribution of the stress in the solid for different time moments. It confirms that the stress decays within a short distance from the cavity surface and decreases quickly with time. Note the curve at $t=0$. It shows that the main decrease in the initial stress distribution occurs within a distance of the cavity diameter from the cavity surface.
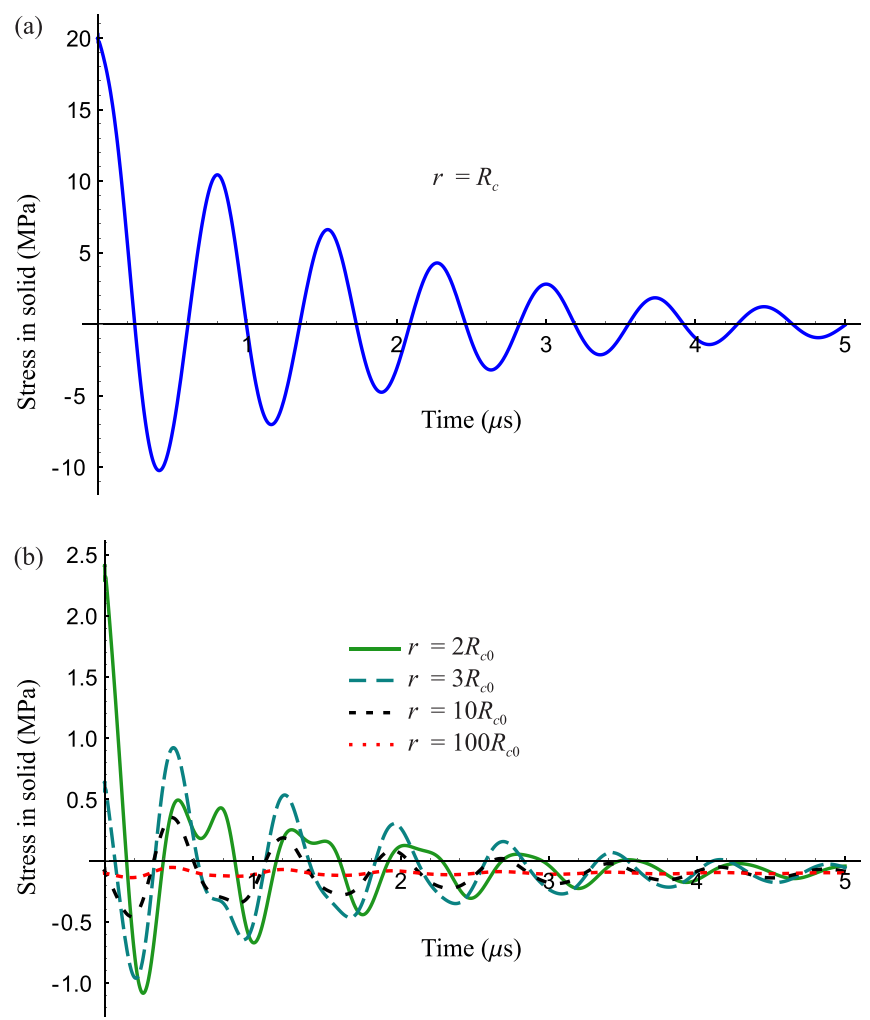

FIG. 5. Stress in the solid as a function of time at different spatial points. The stress at $r=R_{c}$ is shown in the separate plot because of a great difference in magnitude. 

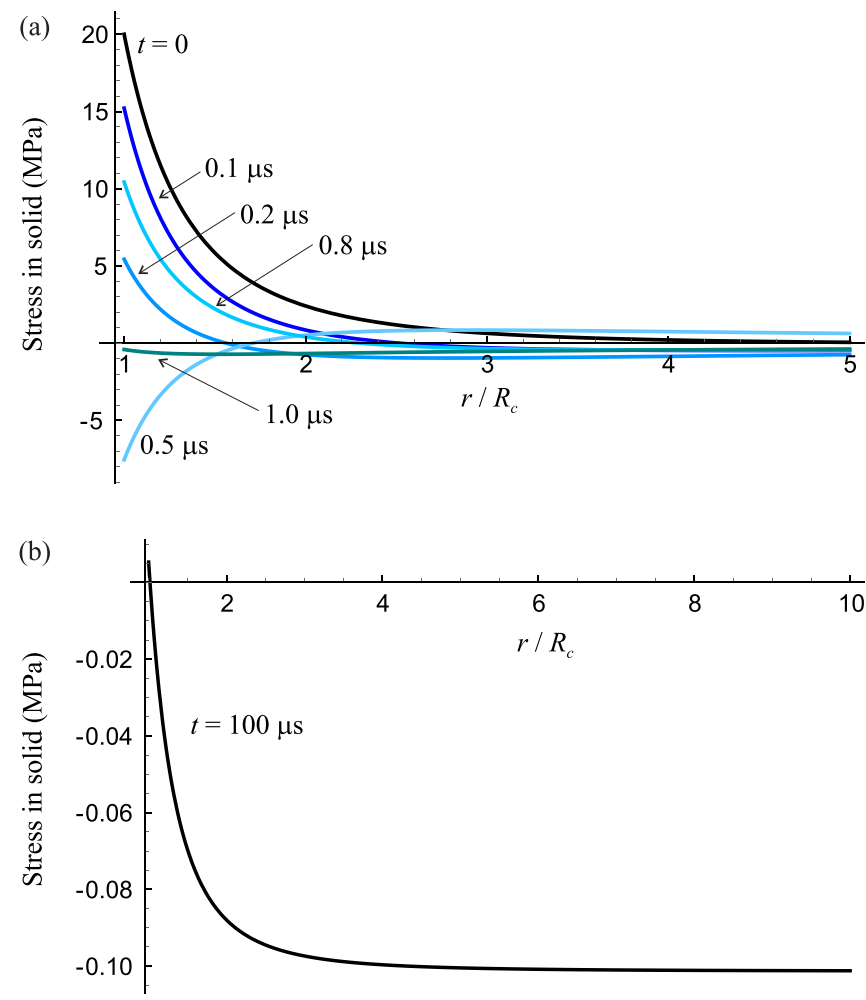

FIG. 6. Spatial distribution of the stress in the solid for different time moments. For $r \rightarrow \infty$, the stress tends to $-P_{\infty}=-101.3 \mathrm{kPa}$. The stress at $t=100 \mu \mathrm{s}$ is shown in a separate plot because of a great difference in magnitude.

Finally, Fig. 7 demonstrates peak positive and negative values of the stress in the solid as a function of the distance from the cavity surface. It also shows that the stress undergoes a considerable gradient only near the cavity surface. The upper curve in Fig. 7 is of special interest because it allows one to estimate the peak negative pressure that is created inside neighboring cavities due to the cavitation process in the cavity under consideration. This problem is considered below. Equation (35) shows that the spatial dependence of the stress in the solid is given by the terms $1 / r$ and $1 / r^{3}$. This fact suggests that the upper curve in Fig. 7 should have the same spatial

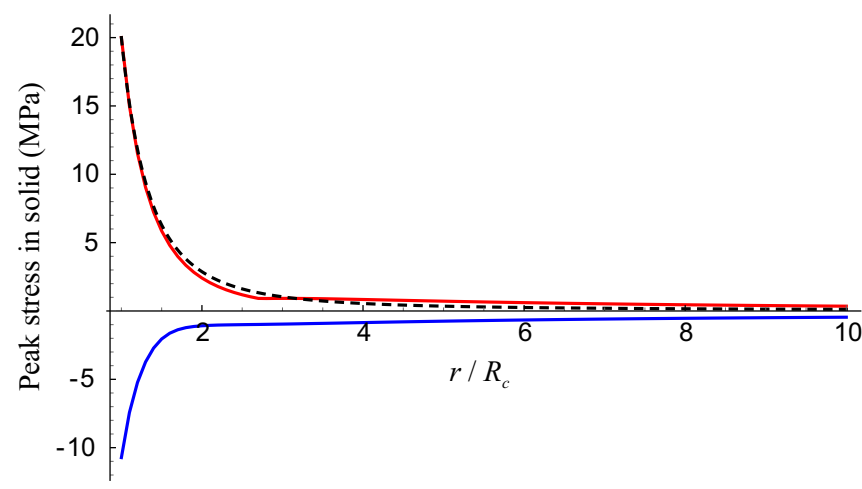

FIG. 7. Peak positive and negative values of the stress in the solid as a function of the distance from the cavity surface. The dashed curve shows the best fit to the upper curve, which is given by Eq. (69).
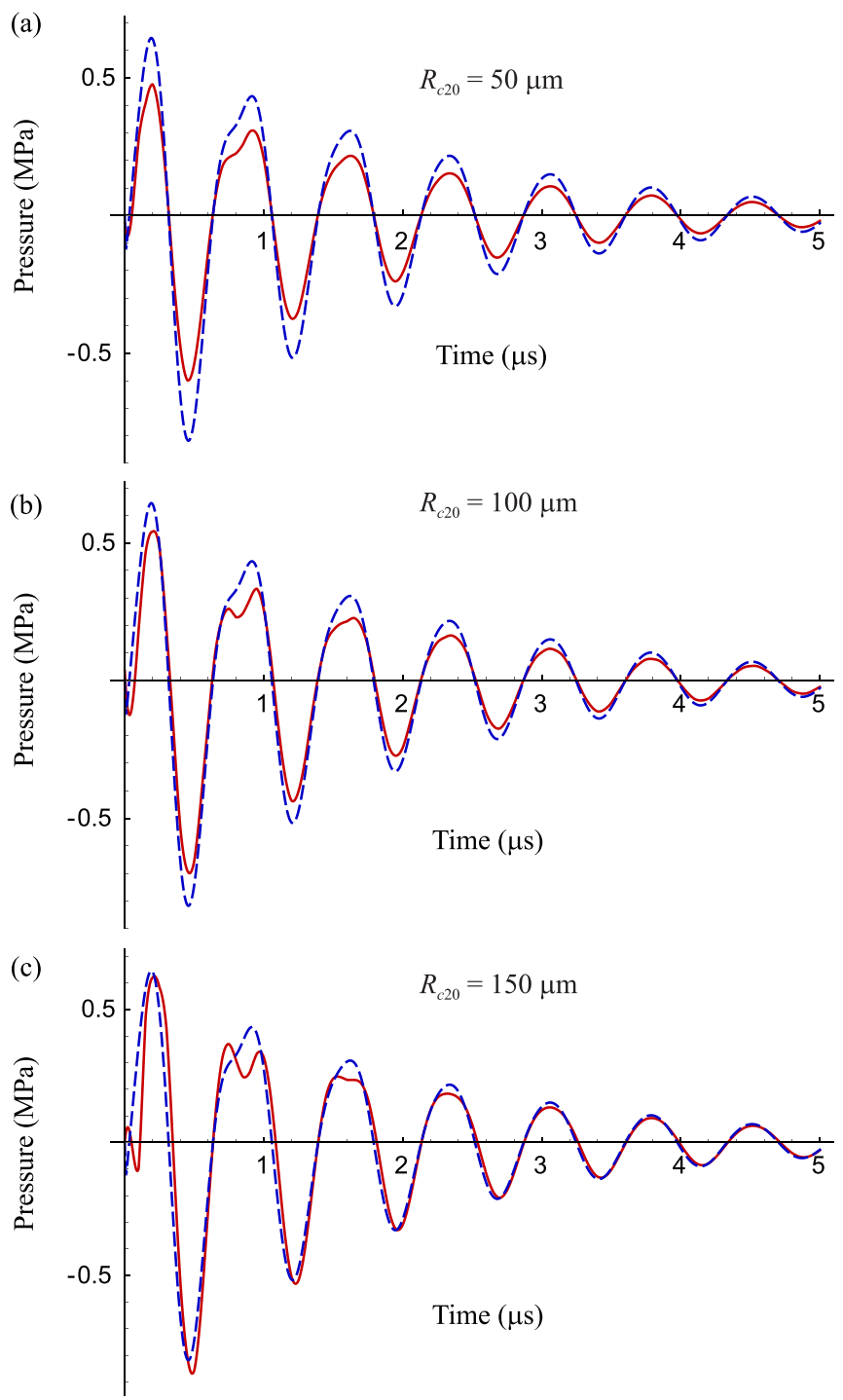

FIG. 8. Disturbance of the liquid pressure at the center of the second cavity at different radii of the second cavity. $R_{c 10}=100 \mu \mathrm{m}$, $d=500 \mu \mathrm{m}$. The solid curves show the pressure at the center of the second cavity. The dashed curves show the pressure that is produced at the surface of the second cavity by the emitted wave from the first cavity. The second half period of the pressure disturbance can trigger the nucleation in the second cavity, increasing the magnitude of the background negative pressure within the second cavity.

dependence. Considering that the peak positive stress at the cavity surface is equal to $-P_{l 0}$, the spatial behavior of the upper curve in Fig. 7 can be approximated by the following equation:

$$
\tau_{+}(r)=-P_{l 0} \frac{R_{c 0}^{3}}{r^{3}}+C P_{l 0}\left(\frac{R_{c 0}^{3}}{r^{3}}-\frac{R_{c 0}}{r}\right),
$$

where $C$ is a constant that can be found by fitting the upper curve in Fig. 7. Equation (69) gives the best fit to the upper curve in Fig. 7 at $C=0.0566$. This fit is shown by the dashed curve.

Figures 8-10 present results obtained by the theory developed in Sec. II B. Figure 8 shows the disturbance of the liquid 


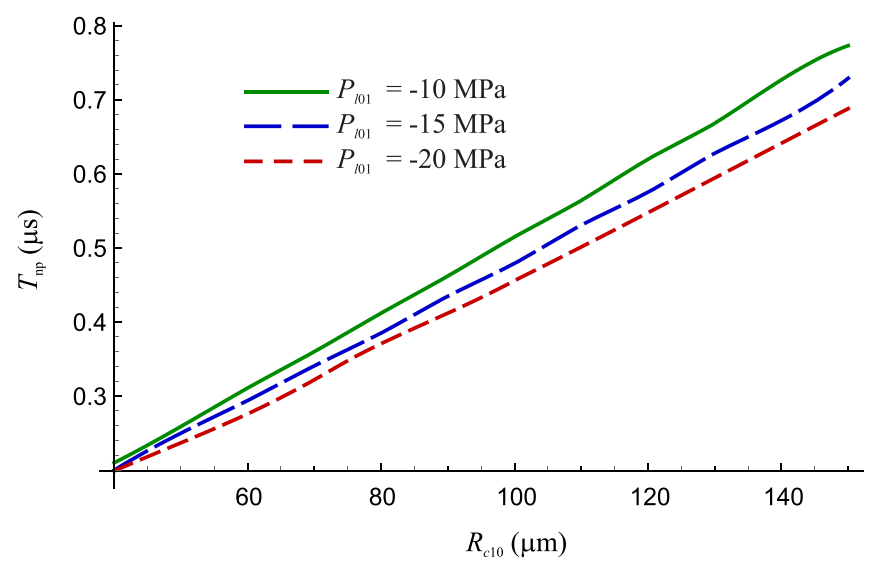

FIG. 9. The time position of the first negative peak of the pressure disturbance at the center of the second cavity as a function of the equilibrium radius of the first cavity $R_{c 10}$ for different values of the initial negative pressure within the first cavity $P_{l 01} . R_{c 20}=50 \mu \mathrm{m}$, $d=500 \mu \mathrm{m}$.

pressure at the center of the second cavity $\left(r_{2}=0\right)$ that is caused by the cavitation process in the first cavity. It is assumed that $R_{c 10}=100 \mu \mathrm{m}$ and $d=500 \mu \mathrm{m}$. The radius of the second cavity, $R_{c 20}$, is varied. The solid curves show the pressure at the center of the second cavity. For comparison, the dashed curves show the pressure that is produced at the surface of the second cavity by the emitted wave from the first cavity. As one can see, the pressure amplitude inside the second cavity is not considerably different from that on the cavity surface. As the radius of the second cavity is reduced, the amplitude of the internal pressure decreases. However, the calculations show that the internal pressure practically stops decreasing when $R_{c 20}$ becomes smaller than $50 \mu \mathrm{m}$. The maximum negative pressure is always reached during the second half period of the pressure disturbance. The magnitude of this pressure decreases with the distance between the cavities, as it must, because the amplitude of the wave produced by the first cavity decays with distance; see Fig. 7.

There is a point to be discussed. We set $R_{c 10} / d=0.2$. Formally, this value is not much smaller than unity. Nevertheless,

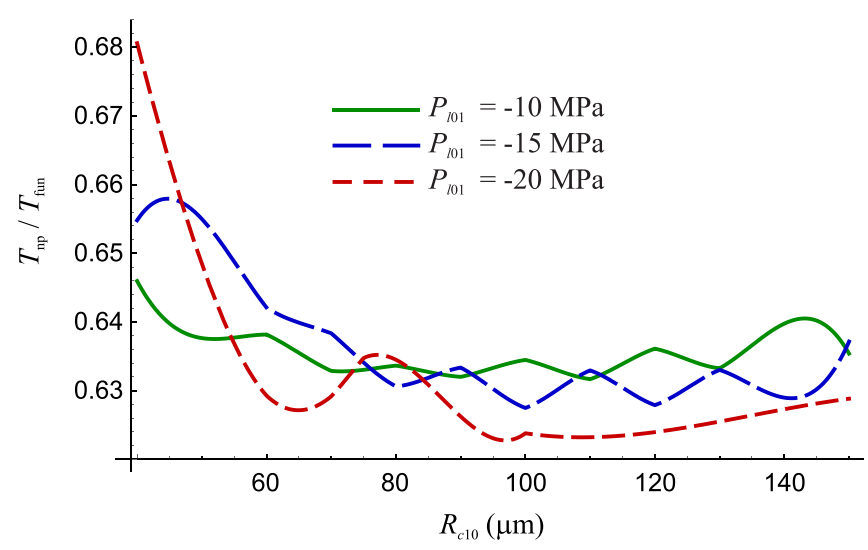

FIG. 10. The ratio of the time position of the first negative peak to the period of the fundamental frequency component of the disturbance wave produced by the first cavity. The parameters are as in Fig. 9. this value still is relatively small so one can expect that the expressions derived in Sec. II B remain dominant at this value and hence have a governing effect on the situation. Therefore, one can expect that the predictions presented in Fig. 8 are correct at least qualitatively. Quantitatively, it is reasonable to expect that they show a correct order of the represented quantities, especially if we take into consideration that an increase in the separation distance only changes the amplitude of the pressure disturbance, changing nothing in the qualitative sense.

It is interesting to consider the results presented in Fig. 8 in the context of experimental data reported by Wheeler and Stroock [27]. In their paper, a microfluidic system is described that was developed to imitate the main attributes of transpiration in plants. This system, called "synthetic tree" formed a microfluidic network of liquid-filled voids in a synthetic hydrogel. Wheeler and Stroock [27] measured the probability of cavitation as a function of negative pressure in the voids; see Fig. 2 in their paper. They found that the probability increased sharply when the pressure dropped below $-20 \mathrm{MPa}$. Considering these observations, let us assume that the background negative pressure within the second cavity is slightly above $-20 \mathrm{MPa}$. Then it follows from Fig. 8 that during the second half period of the pressure disturbance, the total negative pressure within the second cavity may drop below $-20 \mathrm{MPa}$, which in turn may trigger the nucleation in the second cavity. It is also interesting to note that the nucleation is predicted to occur with a time delay with respect to the cavitation process in the first cavity. This delay is determined by the fact that the nucleation is triggered by the second half period of the pressure disturbance rather than by the time of wave propagation between the cavities. This latter is negligible as the sound wavelength is large compared to the distance between the cavities.

The time position of the first negative peak, which will be denoted by $T_{\mathrm{np}}$, can be used as measure of the nucleation delay. $T_{\mathrm{np}}$ is determined by the oscillation frequency of the wave emitted by the first cavity. It was shown in Ref. [19] that this frequency depends on the cavity radius, $R_{c 10}$, and the initial negative pressure within the cavity, $P_{l 01}$. To give an insight into this problem, Fig. 9 shows the time position of the first negative peak as a function of $R_{c 10}$ for three values of $P_{l 01}$. It is assumed that $R_{c 20}=50 \mu \mathrm{m}$ and $d=500 \mu \mathrm{m}$. As one can see, $T_{\mathrm{np}}$, and hence the delay of the nucleation in the second cavity with respect to the cavitation process in the first cavity increases with increasing $R_{c 10}$ and decreasing $P_{l 01}$.

Finally, Fig. 10 shows the ratio $T_{\mathrm{np}} / T_{\text {fun }}$ as a function of $R_{c 10}$, where $T_{\text {fun }}$ is the period of the fundamental frequency component of the disturbance wave produced by the first cavity. As one can see, this ratio changes in relatively narrow limits with changing $R_{c 10}$. One can expect that in experiments, an average delay of about $0.635 T_{\text {fun }}$ should be observed.

\section{CONCLUSION}

The subject of this study is the dynamics of a cavitation bubble in a spherical liquid-filled cavity surrounded by an infinite elastic solid. Two objectives have been pursued. The first was to derive equations for the velocity and pressure fields throughout the liquid filling the cavity and equations 
for the stress and strain fields throughout the solid medium surrounding the cavity. The second objective was to apply the above equations to ascertain if the cavitation process in one cavity can trigger the nucleation in a neighboring cavity. To this end, a second cavity without a bubble was considered. Equations were derived to calculate the disturbance of the liquid pressure within the second cavity, assuming this disturbance to be caused by the cavitation process in the first cavity.

In order to analyze the pressure behavior within the liquid and the stress behavior within the solid, numerical calculations have been first performed for a single cavity. It has been shown that initially the pressure is the same throughout the liquid. When a cavitation bubble appears, a strong pressure difference arises between the bubble wall and the cavity surface. This difference is smoothed in time and eventually, when the bubble radius reaches an equilibrium value, the pressure again becomes uniform throughout the liquid. For the normal stress in the solid, it was found that the main drop in the initial stress distribution occurs within a distance of the cavity diameter from the cavity surface. The stress oscillates due to the oscillation of the cavity surface and the amplitude of the stress oscillation decreases quickly with time and with the distance from the cavity surface.

A second cavity without a bubble has been then included in numerical calculations. The pressure disturbance produced by the emitted wave from the first cavity at the center of the second cavity was calculated for different radii of the second cavity. It was shown that during the second half period of the pressure disturbance, the magnitude of the background negative pressure within the second cavity was increased. According to experimental results reported in Ref. [26], the above effect can trigger the nucleation in the second cavity.

\section{ACKNOWLEDGMENT}

This research has received funding from the European Research Council under the European Union's Seventh Framework Programme (FP7/2007-2013)/ERC Grant Agreement No. 614655 "Bubbleboost."
[1] E. Roedder and R. J. Bodnar, Annu. Rev. Earth Planet. Sci. 8, 263 (1980).

[2] D. Marti, Y. Krüger, D. Fleitmann, M. Frenz, and J. Rička, Fluid Phase Equilib. 314, 13 (2012).

[3] G. W. Scherer and D. M. Smith, J. Non-Cryst. Solids 189, 197 (1995).

[4] D. Or and M. Tuller, Water Resour. Res. 38, 1061 (2002).

[5] O. Vincent, D. A. Sessoms, E. J. Huber, J. Guioth, and A. D. Stroock, Phys. Rev. Lett. 113, 134501 (2014).

[6] O. Vincent, P. Marmottant, S. R. Gonzalez-Avila, K. Ando, and C.-D. Ohl, Soft Matter 10, 1455 (2014).

[7] M. T. Tyree and M. A. Dixon, Plant Physiol. 72, 1094 (1983).

[8] M. T. Tyree and J. S. Sperry, Annu. Rev. Plant. Physiol. Plant Mol. Biol. 40, 19 (1989).

[9] H. Cochard, C. R, Phys. 7, 1018 (2006).

[10] X. Noblin, N. O. Rojas, J. Westbrook, C. Llorens, M. Argentina, and J. Dumais, Science 335, 1322 (2012).

[11] A. D. Stroock, V. V. Pagay, M. A. Zwieniecki, and N. M. Holbrook, Annu. Rev. Fluid Mech. 46, 615 (2014).

[12] M. Larter, T. J. Brodribb, S. Pfautsch, R. Burlett, and H. Cochard, Plant Physiol. 168, 804 (2015).

[13] K. H. Jensen, H. Bruus, N. M. Holbrook, J. Liesche, A. Schulz, and M. A. Zwieniecki, Rev. Mod. Phys. 88, 035007 (2016).
[14] O. Vincent, P. Marmottant, P. A. Quinto-Su, and C.-D. Ohl, Phys. Rev. Lett. 108, 184502 (2012).

[15] O. Vincent and P. Marmottant, J. Fluid Mech. 827, 194 (2017).

[16] S. Mayr and S. Rosner, Tree Physiol. 31, 59 (2011).

[17] T. J. Brodribb and H. Cochard, Plant Physiol. 149, 575 (2009).

[18] B. Choat, S. Jansen, T. J. Brodribb, H. Cochard, S. Delzon, R. Bhaskar, S. J. Bucci, T. S. Feild, S. M. Gleason, U. G. Hacke et al., Nature 491, 752 (2012).

[19] A. A. Doinikov, B. Dollet, and P. Marmottant, Phys. Rev. E 97, 013108 (2018).

[20] Q. X. Wang, Phys. Fluids 29, 072101 (2017).

[21] C. Drysdale, A. A. Doinikov, and P. Marmottant, Phys. Rev. E 95, 053104 (2017).

[22] F. R. Gilmore, Hydrodynamics Laboratory Report No. 26-4 (California Institute of Technology, Pasadena, CA, 1952).

[23] L. D. Landau and E. M. Lifshitz, Theory of Elasticity (Pergamon Press, Oxford, 1970).

[24] L. D. Landau and E. M. Lifshitz, Fluid Mechanics (Pergamon Press, Oxford, 1987).

[25] G. Arfken, Mathematical Methods for Physicists (Academic Press, San Diego, 1985).

[26] M. Abramowitz and I. N. Stegun, Handbook of Mathematical Functions (Dover Publications, New York, 1965).

[27] T. D. Wheeler and A. D. Stroock, Nature 455, 208 (2008). 